\title{
Molecular Basis of Phenylketonuria: From Genotype to Clinical Management
}

\author{
Johannes Zschocke \\ Division of Human Genetics, Medical University Innsbruck, Innsbruck, Austria
}

\section{Key Words}

Phenylketonuria - Mutation analysis • Tetrahydrobiopterin • PAH gene

\begin{abstract}
In the age of neonatal screening and the presymptomatic treatment of individuals with phenylketonuria, disease definition has shifted from a set of clinical symptoms observed in untreated patients to an inherited predisposition that requires dietary intervention to avoid brain damage and intellectual impairment. The analysis of correlations between genotype and phenotype must thus be targeted to the need and modalities of preventive treatment. In phenylketonuria, mutation analysis is not a prerequisite to correct diagnosis and therapy including treatment with the cofactor tetrahydrobiopterin $\left(\mathrm{BH}_{4}\right)$. Nevertheless, the functional impact of individual mutations has been extensively investigated over the last years, and there are well-studied correlations between the genotype and various phenotypic aspects. Knowing and understanding the genotype at a very early stage often allows a prediction of the severity of phenylalanine hydroxylase deficiency and provides a better picture of the disease characteristics in the individual patient. This may assist in the clinical management. Identification of homozygosity or compound heterozygosity for null mutations indicates
\end{abstract}

that this patient will not benefit from $\mathrm{BH}_{4}$ treatment, whilst identification of a well-known $\mathrm{BH}_{4}$-sensitive mutation in a patient who has been classified as non-responsive in a $\mathrm{BH}_{4}$ test should be an indication to re-examine the classification and, if necessary, repeat the test. Care must be taken to recognize potential errors in the results of molecular studies, and to interpret them in relation to clinical and biochemical findings in the individual.

Copyright $\odot 2010$ Nestec Ltd., Vevey/S. Karger AG, Base

\section{Introduction}

There are different approaches to the disease called phenylketonuria (PKU, OMIM 261600), and they reflect the history of its discovery and metabolic, enzymatic and molecular characterization. The story of PKU started with Asbjørn Følling who was confronted with unexplained mental retardation in two siblings. He succeeded in linking the particular clinical phenotype, i.e. mental retardation, unusual body odor, and a few other features, to a specific metabolic phenotype, i.e. abnormal urinary ferric chloride test caused by the presence of phenylpyruvate in the urine [1]. The term phenylketonuria was coined by Penrose [2] and relates to a more exact characterization of the metabolic findings. In 1947,

\section{KARGER}

Fax +4161306 1234

E-Mail karger@karger.ch

www.karger.com (c) 2010 Nestec Ltd., Vevey/S. Karger AG, Basel

0517-8606/10/0682-0048\$26.00/0

Accessible online at:

www.karger.com/ane
Prof. Johannes Zschocke

Division of Human Genetics, Medical University Innsbruck

Schöpfstrasse 41, AT-6020 Innsbruck (Austria)

Tel. +43 5129003 70500, Fax +43 512900373500

E-Mail johannes.zschocke@i-med.ac.at 
Jervis characterized the enzymatic phenotype by pinpointing the metabolic error to the enzyme phenylalanine hydroxylase (PAH) [3]. The successful treatment devised by Bickel et al. [4] and the development of a method for rapid screening in a large number of samples by Guthrie and Susie [5] were the bases of the revolutionary concept of universal neonatal screening which caused a fundamental shift in the clinical phenotype of PKU: away from PKU as a cause of neurological symptoms and intellectual disability to PKU as a genetic trait that necessitates a special low-phenylalanine (phe) diet in order to avoid chronic brain damage. Elevated blood phe concentrations (hyperphenylalaninemia) may also be caused by inherited disorders in the biosynthesis of the cofactor tetrahydrobiopterin $\left(\mathrm{BH}_{4}\right)$, but the term PKU is reserved for PAH deficiency. Finally in the 1980s, the cloning of the human $P A H$ gene [6] and the identification of the first disease-causing mutation [7] marked the beginning of yet another approach to PKU centered on the genotype, the genetic information that determines normal and mutant PAH protein and the development of PKU.

What exactly is meant when one talks about the disease PKU? It is not anymore the clinical phenotype of intellectual disability because this has been largely eradicated by neonatal screening and early initiation of dietary treatment. Rather it is a genetically determined trait that represents a high probability of developing clinical symptoms unless specific therapeutic measures are undertaken. In the age of presymptomatic testing (as practiced by neonatal screening) and preventive medicine, the definition of this genetic disease has thus shifted from clinical symptoms to a genetically determined increased risk for clinical symptoms which may be avoided with certain interventions. In PKU, elevated blood phe concentrations above $360-600 \mu \mathrm{M}$ are taken as a marker for such a risk and represent the limit at which diet is started.

Treatment of PKU has evolved considerably in the last decade. For almost 50 years, there was basically only one therapeutic option: phe restriction through a lowprotein diet in conjunction with supplementation of the other amino acids as well as vitamins, cofactors, and trace elements. This changed with the discovery that administration of the cofactor $\mathrm{BH}_{4}$ causes a reduction in phe concentrations in some patients with PKU [8]. A pharmaceutical preparation of $\mathrm{BH}_{4}$ has now been approved as a treatment option for those patients that respond to its application. Other novel therapeutic strategies including administration of large neutral amino ac- ids that compete with phe for transport at the blood-brain barrier [9], and enzyme replacement therapy with phenylalanine ammonia lyase [10], are currently under investigation.

In consequence of the various aspects discussed above, a genetic approach to the diagnosis of PKU 'from genotype to clinical management' can have three different aims:

(1) Confirmation of the diagnosis PAH deficiency caused by biallelic mutations in the $P A H$ gene, as opposed to primary disorders of $\mathrm{BH}_{4}$ biosynthesis;

(2) Identification of genotypes that predict whether or not treatment will be necessary;

(3) Assessment of treatment modalities, i.e. identification of genotypes that allows the predication of whether or not the patient will be $\mathrm{BH}_{4}$ responsive and/or that provides information on the severity of enzyme deficiency and thus strictness of diet.

The correlation between genotype and phenotype in PAH deficiency with respect to these aims is the focus of this article. Technical aspects that have an impact on the reliability of the molecular results obtained in routine clinical practice will be briefly discussed.

\section{PAH Gene and Mutations}

The PAH gene is located on chromosome 12q24.1 and encompasses approximately $100 \mathrm{~kb}$ of genomic DNA. The gene consists of 13 exons that are translated into a protein of 452 amino acids. More than 550 different disease-causing mutations in the $P A H$ gene have been identified and reported to the $\mathrm{PAH}$ mutation database (www.pahdb.mcgill.ca) [11]; many more may have been detected as part of routine molecular analyses in diagnostic laboratories. More than $80 \%$ of identified mutations are point mutations that have variable effects on transcription and translation; more than half are missense mutations that lead to the exchange of single amino acid residues in the PAH protein. In a diagnostic setting, PCR amplification and sequencing of all exons and adjacent introns achieves a mutation detection rate of $>95-99 \%$. Large deletions that are not recognized by standard PCR-based methods are found on a small proportion $(<2 \%)$ of PKU alleles; they may be detected by multiplex ligation-dependent probe amplification (MLPA) [12]. 


\section{Phenotypes and Genotypes in PAH Deficiency}

A phenotype is defined as an observable characteristic or trait of an organism. With regard to PKU, there are several phenotype levels, as outlined above:

- The clinical phenotype - e.g. intellectual disability, or the need for treatment to avoid intellectual disability;

- The metabolic phenotype - e.g. elevated blood phe concentrations in untreated individuals with PKU;

- The enzymatic phenotype - the residual enzyme function in the liver, or the severity of PAH deficiency.

There is a causal connection between the different phenotypes: the enzymatic phenotype determines the metabolic phenotype, and the metabolic phenotype determines the clinical phenotype. All three phenotypes in PKU can be influenced by therapeutic intervention - the clinical and metabolic phenotypes by diet, the enzymatic phenotypes (in many but not all patients) by $\mathrm{BH}_{4}$ administration.

Independent of treatment and other external factors, there are endogenous differences in the phe tolerance, i.e. the maximum amount of dietary phe that can be taken by a person with PAH deficiency without blood concentrations rising above a certain upper limit. Traditionally, a distinction is made between PKU that requires treatment, and mild hyperphenylalaninemia (MHP) which does not [13]. There are differences in opinion about the exact blood phe concentration that represents the border between PKU and MHP; most recommendations regard concentrations up to $600 \mu \mathrm{M}$ as acceptable but treatment in some countries (e.g. the UK) is started when concentrations consistently exceed $360 \mu \mathrm{M}$ [14]. The neuropsychological data behind the recommendations have been reviewed by Albrecht et al. [15] $]^{1}$. In patients receiving treatment, different degrees of severity such as mild, moderate and severe PKU are distinguished. Various criteria are used for such distinctions; most reliable but also most difficult to determine exactly is the phe tolerance at the age of 5 years [16]. For practical purposes, it can be sufficient to distinguish severe (classical) PKU without residual PAH activity from mild PKU with relatively high phe tolerance; moderate PKU is somewhere in between.

\footnotetext{
1 Interestingly, the discussion on when to start treatment has recently been revived in conjunction with the availability of $\mathrm{BH}_{4}$ treatment, which is effective in many individuals with relatively mild PAH deficiency. It is important to be aware of commercial interests and corporate sponsorship with regard to recommendations to start treatment at lower phe concentrations.
}

The endogenous differences in the enzymatic phenotype and the response to $\mathrm{BH}_{4}$ treatment are linked to the specific mutations in the individual genotype. Extensive studies, particularly in the last 10 years, have elucidated the exact functional effects of a large number of mutations. In many instances, residual enzyme function or responsiveness to $\mathrm{BH}_{4}$ can now be explained by the stability, catalytic integrity and other molecular characteristics of the mutant protein [17-19]. There are a few general principles that can be followed when a genotype identified, say, in a neonate with hyperphenylalaninemia is to be used for predicting the expected severity of PAH deficiency (the phe tolerance or how strict the diet will have to be) or the response to $\mathrm{BH}_{4}$.

(1) Function dominates over non-function. PAH activity in a (compound) heterozygous individual is determined by the allele that codes for the protein with higher enzyme function [20].

(2) A distinction is made between null mutations that completely remove $\mathrm{PAH}$ function and mutations with variable residual activity. By definition, null mutations are not associated with $\mathrm{BH}_{4}$ responsiveness.

(3) Generally, nonsense mutations that insert an early stop codon, frameshift insertions and deletions, and most splice mutations, are null mutations.

(4) Some missense mutations are null mutations but many retain variable function; those with residual activity may or may not be $\mathrm{BH}_{4}$ responsive [21].

(5) PKU patients with homozygosity or compound heterozygosity for null mutations will have severe (classical) PKU with very low phe tolerance, and will not respond to treatment with $\mathrm{BH}_{4}$.

(6)In PKU patients with compound heterozygosity for a null mutation and a mutation with residual function, disease severity and $\mathrm{BH}_{4}$ sensitivity are determined by the latter mutation (functional hemizygosity) [22, 23].

These rules have to be used with a degree of caution since other factors in addition to mutations identified in the $P A H$ gene may influence the individual phe tolerance. There may also be other factors that influence the effects of high phe concentrations on cognitive performance and neurological symptoms; however, the presence of such factors has not been proven and thus cannot be taken into consideration for treatment decisions. 


\section{PKU Mutation Analysis in Clinical Practice}

Mutation analyses are not a prerequisite for optimal treatment of PKU patients as this is based on the regular measurement of phe concentrations and titration of dietary protein accordingly. Mutation analyses are not required for determination of $\mathrm{BH}_{4}$ responsiveness either as not all patients with the same genotype appear to be equally responsive, and the interaction of two different mutations may be difficult to predict. Nevertheless, knowledge of the genotype may help in the assessment of potential $\mathrm{BH}_{4}$ responsiveness and long-term management.

The primary diagnosis of PKU is based on the identification of consistently elevated phe concentrations in blood, and the exclusion, by biochemical tests, of primary deficiencies in the biosynthesis or recycling of the cofactor $\mathrm{BH}_{4}$. Enzyme analyses are not usually possible since $\mathrm{PAH}$ is an exclusively hepatic enzyme. Molecular analysis is not usually necessary to confirm the diagnosis; however, knowing and understanding the genotype at a very early stage often allows a prediction of the severity of PAH deficiency and provides a better picture of the biological characteristics of the disease in the individual patient. This may be useful in the actual clinical management. In classical (severe) PKU, for example, it can be difficult to avoid marked fluctuations in phe values and a rise above the upper therapeutic limit, e.g. during an intercurrent illness [24]. In contrast, similar fluctuations in mild forms of PKU are more likely caused by insufficient adherence to the diet and may be an indication to reassess possible reasons for poor compliance with treatment recommendations. Identification of homozygosity or compound heterozygosity for null mutations indicates that this patient will not benefit from $\mathrm{BH}_{4}$ treatment, whilst identification of a wellknown $\mathrm{BH}_{4}$-sensitive mutation in a patient who has been classified as non-responsive in a $\mathrm{BH}_{4}$ test should be an indication to re-examine the classification and, if necessary, repeat the test. It is important to realize that $\mathrm{BH}_{4}$ sensitivity is a phenotypic trait that is defined by the individual response to $\mathrm{BH}_{4}$, and it is not an obligatory requirement to know the genotype even if it clarifies the underlying biology in the individual case and may help to save effort and money in the diagnostic workup.

PKU mutation analysis is sometimes requested by partners of individuals with PKU or confirmed carriers for PKU mutations in order to assess the likelihood of an affected child. Some couples choose not to have children when there is a high risk of $25 \%$ or even $50 \%$ that a future child will have PKU. In principle, it is easily possible to carry out prenatal diagnosis for PKU by chorionic villus biopsy/amniocentesis and analysis of the familial mutations if they are known. However, termination of pregnancy because of PKU in the expected child is not an option in many countries as the condition is well treatable and has an excellent prognosis. Predictive mutation testing in a family is therefore of limited value. Even if the mutations in a family are known, DNA analysis is not regarded as the method of choice for confirmation of the diagnosis in a neonate as the biochemical test provides the same result but is easier, faster, and cheaper.

\section{Quality of PKU Mutation Analysis}

Mutation analyses have become a normal component in the diagnostic workup of patients with PKU, but limited consideration is often given to the possibility that the reported results may in fact be incorrect. However, any laboratory method may go wrong for a variety of reasons; from sample mix-up to failure to recognize a relevant abnormality. This is especially true for generally complicated and non-automated mutation studies. Correct interpretation of the results requires a special expertise which may not be available in laboratories that offer molecular analyses for a large number of genes for commercial reasons. Unfortunately, errors that have occurred may go unnoticed since DNA studies are relatively costly and are not easily repeated.

An annual External Quality Assessment scheme for PKU mutation analysis instigated in 2004 has given rather disconcerting results in this respect [25]. Participating laboratories received three DNA samples for either diagnostic mutation analysis or carrier testing. Each year, a number of laboratories have failed to determine the correct genotype. Sometimes this was explained by methodical limitations, but each year there has been at least one laboratory that missed a mutation despite using an appropriate technique. Indeed, in one year almost $8 \%$ of all reports from laboratories that used comprehensive mutation detection methods, such as sequencing of all exons of the $P A H$ gene, contained incorrect genotypes. There have also been serious deficiencies in the interpretation of genotype data; in one instance, several laboratories misclassified an MHP mutation (associated with the mild form of PAH deficiency that does not require treatment), and some even offered prenatal diagnosis although an 'affected' child would have been completely healthy with regard to PKU. Molecular diagnostic services should only be provided by laboratories that are 
familiar with genotype-phenotype correlations in the respective disorders.

A few general criteria may guide the referring clinician in choosing a laboratory that provides high-quality mutation analyses. Accreditation and/or participation in External Quality Assessment schemes should indicate that a laboratory has implemented strategies to recognize and avoid errors. Reports may be more likely to reflect up-todate knowledge if the laboratory concentrates on a limited number of tests related to areas of special expertise (possibly documented by publications in the field) and does not offer a large portfolio of molecular tests for commercial reasons. Finally, reports should contain key elements including indication for the test, method employed (if necessary with the sensitivity of the test), exact description of the results using standard nomenclature, and a detailed interpretation in view of the clinical question. There should be transparency in the costs since they may vary considerably between laboratories even within the same country.

\section{Conclusions}

Although mutation analyses are not absolutely essential in the management of patients with PKU, they help in understanding the molecular characteristics of PAH deficiency in the individual patient and assist in treatment decisions. They are useful in the management of all patients with PKU provided that the necessary expertise to interpret the results correctly is available, and the costs of DNA studies are not prohibitive.

\section{References}

1 Følling A: Über Ausscheidung von Phenylbrenztraubensäure im Harn als Stoffwechselanomalie in Verbindung mit Imbezillität. Hoppe Seylers Z Physiol Chem 1934;227: 169-176.

2 Penrose LS: Inheritance of phenylpyruvic amentia (phenylketonuria). Lancet 1935; ii:192-194.

-3 Jervis GA: Studies on phenylpyruvic oligophrenia. The position of the metabolic error. J Biol Chem 1947;169:651-656.

-4 Bickel H, Gerrard J, Hickmans EM: Influence of phenylalanine intake on phenylketonuria. Lancet 1953;ii:812-823.

5 Guthrie R, Susie A: A simple phenylalanine method for detecting phenylketonuria in large populations of newborn infants. Pediatrics 1963;32:338-343.

6 Woo SLC, Lidsky AS, Guttler F, et al: Cloned human phenylalanine hydroxylase gene allows prenatal diagnosis and carrier detection of classical phenylketonuria. Nature 1983; 306:151-155.

7 DiLella AG, Marvit J, Lidsky AS, et al: Tight linkage between a splicing mutation and a specific DNA haplotype in phenylketonuria. Nature 1986;322:799-803.

8 Kure S, Hou DC, Ohura T, et al: Tetrahydrobiopterin-responsive phenylalanine hydroxylase deficiency. J Pediatr 1999;135:375-378.

$\checkmark 9$ Rocha JC, Martel F: Large neutral amino acids supplementation in phenylketonuric patients. J Inherit Metab Dis 2009;32:472-480.

$\checkmark 10$ Sarkissian CN, Shao Z, Blain F, et al: A different approach to treatment of phenylketonuria: phenylalanine degradation with recombinant phenylalanine ammonia lyase. Proc Natl Acad Sci USA 1999;96:2339-2344.
- 11 Scriver CR, Hurtubise M, Konecki D, et al: PAHdb 2003: what a locus-specific knowledgebase can do. Hum Mutat 2003;21:333344.

12 Birk Møller L, Nygren AO, Scott P, et al: Low proportion of whole exon deletions causing phenylketonuria in Denmark and Germany. Hum Mutat 2007;28:207.

13 Güttler F, Guldberg P: The influence of mutations of enzyme activity and phenylalanine tolerance in phenylalanine hydroxylase deficiency. Eur J Pediatr 1996;155(suppl 1):S6S10.

14 Schweitzer-Krantz S, Burgard P: Survey of national guidelines for the treatment of phenylketonuria. Eur J Pediatr 2000;159(suppl 2):S70-S73.

15 Albrecht J, Garbade SF, Burgard P: Neuropsychological speed tests and blood phenylalanine levels in patients with phenylketonuria: a meta-analysis. Neurosci Biobehav Rev 2009;33:414-421.

16 Güttler F: Hyperphenylalaninemia: diagnosis and classification of the various types of phenylalanine hydroxylase deficiency in childhood. Acta Paediatr Scand Suppl 1980; 280:1-80.

17 Dobrowolski SF, Pey AL, Koch R, et al: Biochemical characterization of mutant phenylalanine hydroxylase enzymes and correlation with clinical presentation in hyperphenylalaninaemic patients. J Inherit Metab Dis 2009;32:10-21.

18 Erlandsen H, Patch MG, Gamez A, et al: Structural studies on phenylalanine hydroxylase and implications toward understanding and treating phenylketonuria. Pediatrics 2003;112:1557-1565.
19 Pey AL, Stricher F, Serrano L, Martinez A: Predicted effects of missense mutations on native-state stability account for phenotypic outcome in phenylketonuria, a paradigm of misfolding diseases. Am J Hum Genet 2007; 81:1006-1024.

20 Zschocke J: Dominant versus recessive: molecular mechanisms in metabolic disease. J Inherit Metab Dis 2008;31:599-618.

21 Langenbeck U, Burgard P, Wendel U, et al: Metabolic phenotypes of phenylketonuria. Kinetic and molecular evaluation of the Blaskovics protein loading test. J Inherit Metab Dis 2009;32:506-513.

22 Guldberg P, Rey F, Zschocke J, et al: A European multicenter study of phenylalanine hydroxylase deficiency: classification of 105 mutations and a general system for genotype-based prediction of metabolic phenotype. Am J Hum Genet 1998;63:71-79.

23 Zschocke J, Graham CA, Stewart FJ, et al: Non-phenylketonuria hyperphenylalaninaemia in Northern Ireland: frequent mutation allows screening and early diagnosis. Hum Mutat 1994;4:114-118.

24 Lichter-Konecki U, Rupp A, Konecki DS, et al: Relation between phenylalanine hydroxylase genotypes and phenotypic parameters of diagnosis and treatment of hyperphenylalaninaemic disorders. German Collaborative Study of PKU. J Inherit Metab Dis 1994;17: 362-365.

25 Zschocke J, Aulehla-Scholz C, Patton S: Quality of diagnostic mutation analyses for phenylketonuria. J Inherit Metab Dis 2008; 31:697-702. 